\title{
RANO Ellingson 2017 Oncology Response Criteria
}

National Cancer Institute

\section{Source}

National Cancer Institute. RANO Ellingson 2017 Oncology Response Criteria. NCI

Thesaurus. Code C158262.

Modified Criteria for Radiographic Response Assessment in Glioblastoma Clinical Trials (Benjamin M. Ellingson, Patrick Y. Wen, Timothy F. Cloughesy. Modified Criteria for Radiographic Response Assessment in Glioblastoma Clinical Trials. Neurotherapeutics (2017) 14:307-320). 